\title{
Percepções de estudantes do ensino superior sobre o feedback docente e desempenho acadêmico ${ }^{1}$
}

\author{
Juana de Carvalho Ramos Silval \\ Carolina Fernandes Carvalho"
}

RESUMO

$\mathrm{Na}$ literatura, o feedback docente surge como uma ferramenta necessária à aprendizagem. No entanto, a percepção é a de que o feedback que o estudante constrói interfere em sua eficácia. Esta investigação foca-se na visão de estudantes do ensino superior sobre ofeedback docente e no consequente impacto sobre o desempenho acadêmico. Para tal, aplicou-se um questionário on-line, baseado em duas escalas de percepção de feedback docente, a 773 estudantes de licenciatura de Institutos Federais. Os resultados revelaram qualidades psicométricas aceitáveis do instrumento. Verificou-se, ainda, que, quanto maior a utilização de práticas eficazes de feedback docente, maior a valorização deste por parte dos estudantes e melhor o desempenho acadêmico. Ao encontro da literatura, constatou-se que os discentes tendem a reconhecer como útil o feedback que se apresenta na forma de uma crítica construtiva, específica e que se afasta das simples classificações.

PALAVRAS-CHAVE

ensino superior; feedback docente; estudantes de ensino superior; desempenho acadêmico.

'Instituto Federal de Educação Ciência e Tecnologia de Brasília, Brasília, DF, Brasil.

"Instituto de Educação da Universidade de Lisboa, Lisboa, Portugal.

1 Este artigo insere-se no âmbito de doutoramento em curso da primeira autora e é financiado pela Fundação para a Ciência e a Tecnologia (FCT) por meio de bolsa individual, com a referência SFRH/BD/143275/2019. 


\title{
STUDENTS' PERCEPTIONS ABOUT PROFESSORS FEEDBACK AND ACADEMIC ACHIEVEMENT
}

\begin{abstract}
In the literature, teacher feedback appears as a necessary tool for learning. However, the student's perception of feedback interferes with its effectiveness. This investigation focuses on the view of higher education students on teacher feedback and its impact on academic performance. To this end, an online questionnaire was applied, based on two scales of perception of teacher feedback, to 773 undergraduate students from Federal Institutes. The results reveal acceptable psychometric qualities of the instrument. It was also found that the greater the use of effective teacher feedback practices, the greater the students' appreciation of it and the better their academic performance. In agreement with the literature, it was found that the students tend to recognize feedback as useful when presented in the form of a constructive, specific criticism and moves away from simple classifications.
\end{abstract}

\section{KEYWORDS}

higher education; teacher feedback; higher education students; academic performance.

\section{PERCEPCIONES DE LOS ESTUDIANTES DE EDUCACIÓN SUPERIOR SOBRE EL FEEDBACK Y EL DESEMPEÑO ACADÉMICO}

\section{RESUMEN}

En la literatura, el feedback del docente aparece como una herramienta necesaria para el aprendizaje. Sin embargo, la percepción que tiene el alumno del feedback interfiere con su eficacia. Esta investigación se centra en la visión de los estudiantes de educación superior sobre el feedback de los docentes y su impacto en el rendimiento académico. Para ello, se aplicó un cuestionario en línea, basado en dos escalas de percepción del feedback docente, a 773 estudiantes de pregrado de Institutos Federales. Los resultados revelan cualidades psicométricas aceptables del instrumento. También se encontró que cuanto mayor es el uso de prácticas efectivas del feedback docente, mayor es la apreciación de la misma por parte de los estudiantes y mejor es su desempeño académico. De acuerdo con la literatura, se encontró que los estudiantes tienden a reconocer como útil el feedback que se presenta en forma de crítica constructiva, específica y se aleja de las clasificaciones simples.

\section{PALABRAS CLAVE}

educación superior; feedback de los professores; estudiantes de educación superior; logro académico. 


\section{INTRODUÇÃO}

Hattie e Timperley (2007) referem-se ao feedback como informações fornecidas por um professor, um colega, pai, mãe, ou qualquer outra pessoa em relação a aspectos de um desempenho ou comportamento. Em suas palavras:

Feedback é conceituado como informação fornecida por um agente (por exemplo, professor, colega, livro, pai, si mesmo, uma experiência) em relação a aspectos de seu desempenho ou compreensão. Um professor ou pai pode fornecer informações corretivas, um colega pode fornecer uma estratégia alternativa, um livro pode fornecer informações para esclarecer ideias, um pai pode fornecer incentivo e um aluno pode procurar a resposta para avaliar a exatidão de um item respondido. O feedback, portanto, é uma "consequência" do desempenho. (Hattie e Timperley, 2007, p. 81, tradução das autoras) ${ }^{1}$.

Portanto, é uma consequência observável de uma ação. Contudo, Butler e Winne (1995) afirmam que o feedback pode ser interno ou externo. O primeiro refere-se à forma como os sujeitos, no caso os estudantes, autorregulam seus pensamentos, seus comportamentos e suas emoções. O segundo auxilia o discente a regular sua aprendizagem, partindo das indicações fornecidas por um agente externo. Em ambos os casos, "o moderador mais poderoso que melhora o desempenho é o feedback" (Hattie, 1992, p. 9, tradução das autoras) ${ }^{2}$.

Alguns anos mais tarde, em colaboração com Timperley, acrescentou-se à ideia inicial de Hattie outra complementar: "feedback é uma das influências mais poderosas na aprendizagem e desempenho, mas esse impacto pode ser positivo ou negativo" (Hattie e Timperley, 2007, p. 81, tradução das autoras) ${ }^{3}$. Em outras palavras, o feedback do professor pode ter um sentido diferente do desejado, nomeadamente, quando não gera uma consciencialização valiosa para o aprendiz de seus desempenhos, por não evidenciar as dissonâncias entre o resultado pretendido e o conquistado. Assim, o feedback docente pode incentivar uma mudança, apontar comportamentos adequados, motivando o estudante a repeti-los ou a modificá-los, no sentido de se aproximar do desejado e possibilitar uma conscientização acerca da aprendizagem - contribuição valiosa para a transformação de seus desempenhos (Collins, 2004; Sadler, 2010). Entretanto, esse estímulo do professor pode, também, gerar comportamentos de desânimo, frustração e abandono, quando não se indica um caminho claro para superar desempenhos menos exitosos.

1 Do original: Feedback is conceptualized as information provided by an agent (e.g., teacher, peer, book, parent, self, experience) regarding aspects of one's performance or understanding. A teacher or parent can provide corrective information, a peer can provide an alternative strategy, a book can provide information to clarify ideas, a parent can provide encouragement, and a learner can look up the answer to evaluate the correctness of a response. Feedback thus is a "consequence" of performance.

2 Do original: the most powerful single moderator that enhances achievement is feedback.

3 Do original: feedback is one of the most powerful influences on learning and achievement, but this impact can be either positive or negative. 
Contudo, o feedback é fundamental no processo de ensino-aprendizagem, tanto para os autores behavioristas quanto para os construtivistas ou socioconstrutivistas (Mory, 2004). Para os primeiros, esse retorno do docente pode estar centrado nos resultados - relacionado às atividades de remediação ou de reforço. Também, pode estar associado à ideia de recompensar o esforço - relacionado à melhora da autoestima e, consequentemente, a um maior envolvimento por parte do estudante. Além disso, pode estar mais orientado para os processos - vinculado à qualidade das respostas dos estudantes às estratégias cognitivas ou metacognitivas utilizadas, correspondentes a uma perspectiva cognitivista ou socioconstrutivista (Fernandes, 2005).

Ultrapassada uma concepção behaviorista, na qual o feedback é considerado resposta a estímulos, a perspectiva construtivista ganha novos desenvolvimentos por associar ao estudante um papel ativo, ao interpretar informações recebidas a partir de concepções, crenças e experiências prévias (Mory, 2004; Brookhart, 2008; Carvalho et al. 2014). Com o foco no estudante e nos processos de aprendizagem cognitivos, volitivos e motivacionais - o trabalho passa a ser atribuir sentido ao que realiza. Fazer sentido requer o uso e o controle dos próprios processos de pensamento (Brookhart, 2008). Nessa abordagem construtivista e socioconstrutivista, o professor precisa promover o diálogo com e entre seus estudantes, e estes devem envolver-se ativamente na aprendizagem (Askew e Lodge, 2000). Ao fazê-lo, dão, também, informações ao docente de como está acontecendo a atividade em sala de aula. Assim, o poder do feedback do professor reside em uma dupla dimensão: cognitiva e motivacional (Carvalho et al.,2014). Desse modo, esse retorno torna-se útil por conter informações que o discente pode utilizar, o que pressupõe que ele terá de ser capaz de o ouvir e de o compreender, adotando uma postura ativa, para autoavaliar o que consegue realizar, e, ainda, o que lhe falta para poder tornar-se mais competente em um determinado aspecto ou realizar determinada tarefa.

Estudos como os de Carvalho et al. (2014), realizados com estudantes portugueses, corroboram essa perspectiva ao focar no discente e em seus modos de aprendizagem, reconhecendo o papel ativo do sujeito aprendiz no processo de feedback do professor. Assim, só há feedback eficaz para a aprendizagem quando a informação recebida é entendida pelo estudante, permitindo superar as dificuldades e melhorar ou regular o desempenho (Sadler, 2016). Segundo Sadler (2016), os estudantes deverão aprender a desenvolver comportamentos e atitudes como:

- interpretar o feedback recebido;

- estabelecer relações entre o feedback e as características do trabalho desenvolvido;

- saber como podem melhorar seu trabalho.

Esse movimento de resposta ao feedback do professor é complexo, pois as pessoas reagem de formas distintas ao que, em tese, seria o mesmo feedback, fazendo, assim, variar os efeitos. Para Swaffield (2008), esses efeitos dependem do educando e, também, da relação destes com quem providencia o feedback, no caso o docente.

É nesse sentido que Fernandes (2005) considera ofeedback determinante para ativar os processos cognitivos e metacognitivos dos estudantes, regular e controlar 
as formas de aprendizagem e melhorar a motivação e a autoestima. Para isso, o docente deve ir além da classificação, deve orientar os discentes para a superação acadêmica de forma sistemática, em ações avaliativas ao longo do processo de ensino-aprendizagem. $\mathrm{O}$ autor afirma, ainda, que as orientações devem estimular e motivar os estudantes, reconhecendo progressos, sucessos e ajudando a superar suas dificuldades ou expandir seus conhecimentos e competências. Em outras palavras, os estudantes precisam de um feedback acerca de seus processos e de seus resultados acadêmicos que lhes possibilite aprimorar o desempenho e a aprendizagem.

Hattie e Timperley (2007) desenvolveram um modelo de feedback composto por quatro níveis:

1. a tarefa, ou produto, que transmite informações sobre erros, ou seja, verifica se algo está correto ou incorreto;

2. o processo, que informa sobre a relação entre o que o estudante fez e a qualidade de seu desempenho e sobre possíveis alternativas e estratégias, promovendo a crença na conquista relacionada a estratégias específicas, tipos singulares de esforço que estão sob o controle do estudante;

3. a autorregulação, relacionada à capacidade em autoavaliação ou à confiança para se dedicar mais a uma tarefa, eficaz na medida em que aumenta a autoeficácia;

4. o próprio sujeito, que pode incentivar o fatalismo acadêmico, atribuindo resultados relacionados a características intrínsecas do indivíduo.

De acordo com o modelo, os três primeiros níveis têm um impacto positivo sobre a aprendizagem, e o último seria, em geral, o menos eficaz, podendo, mesmo, ter efeitos negativos no desempenho do sujeito. Porém, como os autores alertam, cada um dos quatro níveis influencia a eficácia do feedback do professor (Hattie e Timperley, 2007).

Em Portugal, Pereira e Flores (2013) realizaram um estudo com 254 estudantes do Ensino Superior com o objetivo de conhecer as perspectivas de discentes sobre os métodos utilizados e ofeedback de professores. Essa pesquisa revelou que os estudantes tendem a valorizar o feedback que percepcionam como crítica construtiva, embora admitam que as consequentes eficácia e utilidade dependem da compreensão das tarefas que lhes estão associadas. Posteriormente, as autoras - em estudos separados (Flores et al., 2014; Pereira et al., 2016) — verificaram que estudantes consideravam o feedback do professor mais relevante e eficaz quando eram usadas metodologias de trabalho centradas no educando, em oposição às abordagens mais tradicionais. Foi constatado, ainda, por meio dessas mesmas pesquisas, que, quando os estudantes universitários são confrontados com formas de avaliação e de prática mais tradicionais, recebem menos feedback visto como eficaz.

Em outro estudo, realizado com 56 estudantes universitários portugueses de educação do primeiro ano, Silva et al. (2019) identificaram que a maior parte dos estudantes (48\%) tem uma percepção positiva de um feedback docente quando o compreende e, somente nessa situação, consideram-no um fator crucial para a aprendizagem. No Brasil, Farias et al. (2015) concluíram, em um estudo com 120 estudantes da Universidade Federal do Espírito Santo, que o feedback é parte es- 
sencial da educação universitária, afirmando, também, que os estudantes tendem a valorizá-lo quando se apresenta na forma de uma crítica construtiva. Entretanto, os discentes admitiram que a eficácia do feedback docente depende da clareza com que é feito e da relação que têm com o professor.

$\mathrm{Na}$ Escócia, o estudo realizado por Carver (2016) na Universidade de Edimburgo com 613 estudantes partiu da premissa, presente na literatura acadêmica, de que a complexidade do feedback estava em desacordo com a forma como os estudantes o entendiam. No entanto, a pesquisa revelou que a prontidão ou o nível de detalhe desse retorno do professor é menos relevante do que a questão de quem o fornece e de qual o motivo para fazê-lo. Esses resultados sugerem uma necessidade de genuidade no feedback, enfatizando a importância da relação professor-estudante: "Há um forte senso neste estudo de uma necessidade de genuinidade no feedback, enfatizando a importância de relacionamentos verdadeiramente nutritivos e cuidadosos entre tutores e estudantes" (Carver, 2016, p. 51, tradução das autoras) ${ }^{4}$. $\mathrm{O}$ autor conclui que os estudantes identificam os benefícios do feedback orientado para a aprendizagem.

Uma investigação com 278 estudantes universitários da Nova Zelândia explorou crenças de educandos sobre o papel e o objetivo do feedback (Brown, Peterson e Yao, 2016). O estudo conclui que essa resposta por parte do docente orienta as próximas etapas do aprendizado e contribui ainda para a autorregulação das aprendizagens, para a autoeficácia e para o desempenho acadêmico.

Um aspecto ainda pouco explorado na investigação é a relação entre professor e estudante quanto às preferências de feedback em função do gênero. Um estudo de Evans e Waring (2010) investigou essa relação, realizado com 108 estudantes aspirantes a futuros professores. Para os autores, um foco no gênero ainda é necessário, pois, embora se saiba pela literatura que as diferenças de gênero influenciam as preferências de aprendizagem e o desempenho nas tarefas e no processamento de informações, ainda não ficou claro se essas diferenças se estendem às predileções de feedback. Em outro trabalho (Monteiro et al., 2019), desenvolvido com 82 estudantes do ensino básico de português, verificou-se uma interação efetiva entre o foco do feedback, o gênero e o desempenho acadêmico, sendo que os rapazes com desempenho elevado apresentaram vantagens.

Assim, em diferentes contextos educativos, desde os estudantes do ensino obrigatório até os do ensino superior, o feedback é um fator fundamental para a aprendizagem, enquanto construção e transformação progressiva de conhecimento. Com essa ferramenta de interação, os alunos têm conhecimento de como devem agir, raciocinar e realizar algo para conseguirem atingir os objetivos acadêmicos (Silva et al., 2019). A literatura indica, ainda, que o feedback do professor é uma ação poderosa, quando se pensa em modificar o desempenho escolar dos estudantes (Hattie, 1992; Mory, 2004; Hattie e Timperley 2007; Brookhart, 2008; Carvalho et al., 2014; Silva et al., 2019), podendo manifestar-se no clima de sala de aula,

4 Do original: There is a strong sense in this study of a need for genuineness in feedback, emphasizing the importance of truly nurturing and caring relationships between tutors and students. 
quando é pautado por aceitação social e consideração positiva, tanto pelos pares quanto pelos professores, favorecendo a comunicação, a aprendizagem e a realização acadêmica (Ferreira, 2019).

A investigação em torno do feedback docente e, em particular, da percepção dos estudantes do ensino superior demanda continuidade de investigar a complexidade associada à eficácia dessa ação nos desempenhos dos aprendizes. Entende-se o desempenho acadêmico como com caráter multidimensional, não se limitando a fatores individuais. Para Vargas (2014), esse desempenho é considerado uma referência sobre a eficiência do processo educativo, tendo em vista que os professores fornecem feedback na esperança de que os estudantes se apropriem deste ativamente, de forma a aprimorar e regular o aprendizado (Brown, Peterson e Yao, 2016).

Investigar o desempenho acadêmico dos estudantes é essencial, por permitir identificar fatores que o afetam, proporcionando informações para a tomada de decisões estratégicas (Brandt, 2017) por parte da instituição de ensino superior, dos professores e de outros setores sociais políticos e econômicos da sociedade (Brandt et al., 2020). Assim:

O conhecimento dos fatores que mais afetam os resultados acadêmicos do corpo discente permitirá, quer às Universidades, quer às diferentes administrações públicas, ter um valioso instrumento de tomada de decisão e de implementação de ações destinadas a melhorar esses resultados, matéria especialmente importante em um contexto de mudança como o atual. (Durán Santomil et al., 2016, p. 152 , tradução das autoras). ${ }^{5}$

Segundo Kuenzer (2005), a educação brasileira apresenta, historicamente, uma dualidade estrutural, caracterizada pela existência de tipos diferentes de escola para classes sociais distintas. Especificamente no ensino superior, os Institutos Federais de Educação, Ciência e Tecnologia surgem como uma opção de inclusão para o público marginalizado do sistema educacional. Para além dessa inserção, associa-se ao viés do universo profissional, possibilitando a emancipação e a transformação social. A lei n. ${ }^{\circ} 11.892$, de 2008 , que originou os Institutos Federais, afirma que a instituição tem como uma de suas finalidades "ofertar educação profissional e tecnológica, em todos os seus níveis e modalidades, formando e qualificando cidadãos com vistas na atuação profissional nos diversos setores da economia, com ênfase no desenvolvimento socioeconômico local, regional e nacional" (Brasil, 2008).

De acordo com Minghelli (2018), os Institutos Federais se constituíram como uma promessa inovadora pelos seguintes fatores: verticalidade educacional;

5 Do original: El conocimiento de los factores que más inciden en los resultados académicos del estudiantado permitirá, tanto a las Universidades como a las diferentes administraciones públicas, disponer de un instrumento valioso para la toma de decisiones y poner en marcha actuaciones tendentes a mejorar dichos resultados, cuestión especialmente importante en un contexto de cambio como el actual. 
integração de saberes científicos, tecnológicos e culturais; certificação de saberes não formais; formação de professores; forte interação com a comunidade, apresentando uma capilaridade e uma adequação aos arranjos produtivos locais. O objetivo era romper com a hierarquização do conhecimento, combinado com a estratificação das classes sociais.

Para isso, intencionou-se a criação de uma matriz institucional que redirecionasse a política educacional ao mesmo tempo que reposicionasse a potencializasse por meio da expansão de unidades de ensino, descentralizando o acesso a um ensino público, gratuito e de qualidade. Essa nova matriz baseava-se nos IF, o centro da política de reestruturação da Rede Federal de Educação Profissional e Tecnológica (Minghelli, 2018).

Significa dizer que os Institutos Federais não são uma escola técnica e, também, não são uma universidade, pois se caracterizam pela matriz marxista da indissociabilidade entre os compromissos de ordem técnica, prática e a compreensão teórica. Os Institutos Federais são o ponto de convergência dos estudos teóricos, políticos e tecnológicos, com o claro objetivo de interpretar o mundo e transformá-lo. (Minghelli, 2018, p. 162).

Concretamente, no presente estudo, procurou-se conhecer as qualidades psicométricas de um questionário elaborado, para avaliar as percepções de estudantes brasileiros de cursos de licenciatura de IF acerca do feedback dos professores. Posteriormente, buscou-se conhecer como essas percepções se relacionam com o desempenho acadêmico, de acordo com o sexo, a idade e a região na qual se estuda.

\section{MÉTODO}

Nesta seção, será descrito o método utilizado nesta investigação. Primeiro, serão apresentados os participantes. Depois, discorrer-se-á sobre os instrumentos e os procedimentos adotados.

\section{PARTICIPANTES}

A população-alvo deste estudo são estudantes que frequentam cursos de licenciatura em Institutos Federais de Educação, Ciência e Tecnologia de diversas regiões do Brasil. Após convite, participaram Institutos das regiões Norte, Sul, Centro-Oeste e Sudeste, que consentiram a liberação e utilização dos endereços eletrônicos dos referidos estudantes para a coleta de dados no primeiro semestre letivo de 2019. A amostra foi voluntária, composta pelos discentes que responderam ao questionário on-line, enviado por e-mail, hospedado na plataforma Survio. Os envios foram feitos conforme listagem de e-mails emitida pelas próprias Instituições, respeitando as orientações da Comissão de Ética do Brasil, que autorizou o presente estudo.

Responderam ao questionário 773 estudantes de cursos de licenciatura de IF. Desse total, $206(26,9 \%)$ informaram não cursar mais a licenciatura, seja 
por conclusão (136 estudantes, 66\%), seja por evasão (70 discentes, 34\%). Esses questionários, somados aos de dois estudantes menores de idade, foram excluídos da pesquisa, o que reduziu o número de participantes para 565 sujeitos. Ainda, foram excluídos 46 participantes que responderam menos de $90 \%$ dos itens do instrumento de recolha de dados. Assim, a amostra final contou com 519 participantes, distribuídos pelos Institutos Federais das regiões Centro-Oeste (A), Sudeste (B e C), Sul (D, E e F) e Norte do país (G). Os nomes dos Institutos foram suprimidos, assegurando o anonimato das instituições e de seus educandos (conforme a Tabela 1).

Tabela 1 - Distribuição de sujeitos por Instituto Federal em ordem decrescente.

\begin{tabular}{l|c|c|c}
\hline Instituto & Região & Respostas & Percentual \\
\hline Instituto Federal $A$ & Centro-Oeste & 304 & 58,6 \\
\hline Instituto Federal $F$ & Sul & 56 & 10,8 \\
\hline Instituto Federal $D$ & Sul & 71 & 13,7 \\
\hline Instituto Federal $G$ & Norte & 62 & 11,9 \\
\hline Instituto Federal $C$ & Sudeste & 14 & 2,7 \\
\hline Instituto Federal $B$ & Sudeste & 9 & 1,7 \\
\hline Instituto Federal $E$ & Sul & 2 & 0,4 \\
\hline Não indicado & - & 1 & 0,2 \\
\hline Total & & 565 & 100 \\
\hline
\end{tabular}

Fonte: Banco de dados da investigação.

Elaborado pelas autoras.

A idade dos estudantes varia dos 18 a 57 anos, o que explica o alto valor do desvio padrão $(\mathrm{DP}=8,83)$, sendo a média de 26,68 anos e a maioria $(69,2 \%)$ do sexo feminino. A distribuição dos estudantes por curso pode ser observada na Tabela 2.

\section{INSTRUMENTOS}

Utilizou-se um questionário on-line autodeclarado, composto por três partes: 1) caracterização dos participantes; 2) desempenho acadêmico; 3) percepções sobre o feedback docente. Para analisar a percepção de feedback docente, fez-se uso de um questionário com 21 itens na forma de escala de classificação tipo likert de 6 pontos, com número equivalente de respostas positivas e negativas, semanticamente opostos $(0$ = discordo totalmente; 6 = concordo totalmente). $\mathrm{O}$ instrumento foi elaborado a partir da associação de duas escalas.

A primeira escala denomina-se Student conceptions of feedback (SCoF I) e foi criada por Irving e Peterson (2006 apud Brown, Peterson e Yao, 2016) para investigar concepções de estudantes sobre propósitos do feedback. Originalmente, foi aplicada a estudantes do ensino médio (SCoF II), tendo sido adaptada para o ensino superior por Brown, Peterson e Yao (2016), o que deu origem à terceira versão da escala 
Tabela 2 - Distribuição da amostra por curso.

\begin{tabular}{l|c|c}
\hline Curso & $\mathrm{n}$ & $\%$ \\
\hline Ciências Agrárias & 3 & 0,6 \\
\hline Ciências da Natureza/Biológicas & 113 & 21,8 \\
\hline Computação & 5 & 1,0 \\
\hline Dança & 42 & 8,1 \\
\hline Educação do Campo & 1 & 0,2 \\
\hline Física & 23 & 4,4 \\
\hline Geografia & 9 & 1,7 \\
\hline Informática & 5 & 1,0 \\
\hline Letras & 16 & 3,1 \\
\hline Letras: Língua Estrangeira & 66 & 12,7 \\
\hline Letras: Língua Portuguesa & 52 & 10,0 \\
\hline Licenciatura para Educação Profissional e Tecnológica & 31 & 6,0 \\
\hline Matemática & 39 & 7,5 \\
\hline Pedagogia & 27 & 5,2 \\
\hline Programação Especial de Formação Pedagógica de Docentes & 9 & 1,7 \\
\hline Química & 78 & 15,0 \\
\hline Total & 519 & 100,0 \\
\hline Fon Bara da & &
\end{tabular}

Fonte: Banco de dados da investigação.

Elaborado pelas autoras.

(SCoF III). Nesta versão, o instrumento contém 42 itens na forma de escala de classificação tipo likert de 6 pontos e mais um item de múltipla escolha. Conta com apenas duas opções negativas (discordo totalmente e discordo principalmente) e quatro opções positivas (concordo ligeiramente, concordo moderadamente, concordo principalmente e concordo totalmente).

Matos (2010) e Matos e Brown (2015) fizeram uma adaptação da escala ScoF III, referente, especificamente, à avaliação para o contexto brasileiro. No entanto, no presente estudo, focou-se no feedback do professor. Todo o instrumento foi traduzido e recebeu a anuência dos autores. Assim, não foram utilizados todos os itens da escala SCoF III, considerando escopo e pertinência dos itens, preocupações com a fadiga dos participantes pela extensão do instrumento e pela necessidade de autorreflexão que o questionário exige. Optou-se apenas pelos itens de maior adequação aos objetivos da investigação, ou seja, os referentes somente ao feedback docente, totalizando a utilização de oito tópicos. Alterou-se também a escala, de forma a haver equivalência entre itens positivos e negativos - discordo totalmente, discordo principalmente, discordo pouco, concordo ligeiramente, concordo moderadamente, concordo totalmente.

A segunda escala é a de Carvalho et al. (2014). Foram utilizados todos os 11 itens dessa escala, alterando somente a de tipo likert de 4 pontos $($ sempre $=3$; 
nunca $=0)$ para a de 6 pontos, descrita no instrumento anterior. Houve adequações linguísticas, considerando que o instrumento foi aplicado originalmente em Portugal $\mathrm{e}$, neste estudo, foi adaptado aos estudantes brasileiros.

O questionário final avaliou concepções e percepções do feedback docente na perspectiva de estudantes, relacionando-as com o desempenho acadêmico autodeclarado em cursos de licenciatura no Brasil. Além das escalas supracitadas, os estudantes foram questionados em relação à média de notas das disciplinas cursadas, o número total de disciplinas já cursadas e o número de matérias que reprovaram até o fim do primeiro semestre letivo de 2019.

\section{PROCEDIMENTOS}

O início da pesquisa começou com o pedido à Comissão de Ética brasileira. Após a aprovação, esclareceu-se a todos os participantes o teor do estudo, e eles informaram consentimento. Posteriormente, o instrumento foi enviado por meio do correio eletrônico dos estudantes, de forma automática, pela plataforma de investigação Survio. A coleta foi realizada durante o mês de julho de 2019, fim do primeiro semestre letivo brasileiro. Esse momento foi escolhido para que os estudantes já tivessem acesso ao feedback e às avaliações acadêmicas. Assim, discentes, incluindo os que ingressaram no primeiro semestre, puderam participar do estudo.

Depois de finalizada a coleta de dados, realizou-se a análise fatorial exploratória (AFE) do instrumento. Os missing values foram imputados pelo método de Hot-Deck Multiple Imputation, como sugerido por Lorenzo-Seva e Van Ginkel (2016). Para avaliar a validade da realização de uma AFE nos 19 itens que conformam o instrumento, observou-se se as variáveis eram homogêneas e se existiam correlações entre os itens. Para avaliar a homogeneidade das variáveis, utilizou-se o critério Kaiser-Meyer-Olkin (KMO), com as categorias de classificação definidas em Howard (2016): valores inferiores a 0,5 são considerados inaceitáveis; valores entre 0,5 e 0,6 pobres; entre 0,6 e 0,7 medíocres, mas aceitáveis; entre 0,7 e 0,8 intermédios; entre 0,8 e 0,9 bons; entre 0,9 e 1 excelentes. Assim, valores próximos a 1 indicam que existem correlações parciais entre os itens e é possível fazer uma AFE. Também, foi utilizado o teste de esfericidade de Bartlett, para avaliar se havia conexão entre os itens. Observou-se um $\mathrm{KMO}=0,92$ e um teste de Bartlett de $\chi^{2}(171)=3.701,6, p<0,001$, uma fatorabilidade da matriz de correlações excelente. Foi possível, portanto, proceder a uma $\mathrm{AFE} \mathrm{com} \mathrm{os} \mathrm{dados} \mathrm{da} \mathrm{amostra.}$

Para as análises, foi utilizado o programa FACTOR 10.10.03 (Lorenzo-Seva e Ferrando, 2006; Ferrando e Lorenzo-Seva, 2019), a fim de definir a estrutura fatorial pelo método Robust Unweighted Least Squaares (RULS). Usou-se, nesse contexto, uma matriz de correlações policóricas, como recomendado para a análise fatorial de escalas de itens ordinais de tipo likert (Holgado-Tello et al., 2010; Asún, Rdz-Navarro e Alvarado, 2015). Uma vez que esperávamos que as dimensões avaliadas estivessem correlacionadas, foi aplicado o método de rotação oblíqua Promin (Lorenzo-Seva e Ferrando, 2013).

Para reter apenas o número mínimo de fatores, foi utilizado o critério Kaiser-Guttman como ponto de partida, isto é, os fatores com autovalores (eigenvalues) superiores a 1 . No entanto, uma vez que esse critério tende a superestimar 
o número de fatores retidos, recorreu-se ao método da implementação ótima de análises paralelas (Optimal implementation of parallel analysis, AP), encontrado em Timmerman e Lorenzo-Seva (2011), para confirmar esses resultados. Nesse método, a percentagem de variância, explicada pelos dados por meio do minimum rank factor analysis, compara-se (pareia-se) com o percentil 95 da variância explicada por um conjunto hipotético de dados de 500 matrizes de correlação de variáveis determinados aleatoriamente. Assim, os fatores com valores de variância explicada inferiores aos calculados nas AP foram descartados (Timmerman e Lorenzo-Seva, 2011).

Finalmente, para determinar se os modelos fatoriais sugeridos representam corretamente os dados, foram calculados índices de ajustamento (utilizando Bootstrap para análises mais robustas), apresentados, a seguir, junto com os valores de corte sugeridos (Schreiber et al., 2006): rácio do qui-quadrado pelos graus de liberdade $\left(\chi^{2} / \mathrm{df} \leq 2\right.$ ou 3$)$; comparative fit index (CFI $\left.\geq 0,95\right)$; Tucker-Lewis index ( $\mathrm{TLI} \geq 0,96$ ); root mean square error of approximation (RMSEA $<0,06$ a $0,08 \mathrm{com}$ intervalo de confiança).

Ainda, avaliou-se a consistência interna dos fatores retidos, utilizando o alfa de Cronbach, sendo valores 0,8 - 0,9 considerados bons e > 0,9 excelentes ( $\mathrm{Ma}-$ rôco, 2014). Calculou-se os scores de cada fator do modelo adotado pela média dos itens que o conformam. Foram medidas as estatísticas descritivas (média e DP). Contabilizou-se os coeficientes de correlação de Pearson $(r)$ entre as variáveis em estudo. Para identificar as diferenças entre grupos, foi utilizado o teste T-Student e a Análise de Variância (Anova). Além disso, foi verificado um índice de repetência, sendo o coeficiente oriundo da relação entre o número de matérias em que o estudante reprovou e o número total de disciplinas já cursadas por ele.

Avaliou-se, também, a validade de critério. Foi analisada a qualidade do ajustamento de um modelo causal, elaborado no software AMOS (vs. 25), e as percepções do feedback do estudante sobre o próprio desempenho acadêmico, utilizando os índices de ajustamento previamente mencionados ( $\chi^{2} / \mathrm{df}$; CFI; TLI; RMSEA).

\section{RESULTADOS}

O baixo índice de resposta dos Institutos $B$ e $C$ decorre de não concederem o acesso aos $e$-mails de seus estudantes para contato direto, mas, sim, terem enviado o instrumento diretamente aos alunos, por meio dos coordenadores de curso. Notou-se que o envio institucional não abrangeu todos os cursos de licenciatura das IF; além disso, esse fato pode ter intimidado os discentes, que possivelmente tiveram receio de seus professores, coordenadores, entre outros terem acesso às respostas. Outra possibilidade para o baixo retorno dos discentes que receberam o instrumento internamente pode dever-se a uma única tentativa de coleta de dados, enquanto o método de envio em massa e automatizado, realizado com a plataforma, encaminha lembretes eletrônicos a cada cinco dias aos sujeitos que ainda não tenham respondido.

Inicialmente, com a realização de $\mathrm{AFE}$, seguindo o critério de Kaiser-Guttman, os resultados alcançados com os 19 itens do instrumento, para avaliar as concepções e percepções dos estudantes em relação ao feedback do docente, sugeriram 
a extração de três fatores que explicam $61,54 \%$ da variância. No entanto, apenas dois apresentaram autovalores superiores à média dos obtidos em uma análise paralela com 500 matrizes correlacionais aleatórias, sugerindo a retenção de três fatores, que explicam $54,80 \%$ da variância.

$\mathrm{Na}$ Tabela 3, resumem-se os pesos fatoriais de cada item para cada um dos fatores e a consistência interna, avaliada pelo alfa de Cronbach (Tabela 4). O modelo é considerado adequado em virtude de apresentar índices de ajustamento excelentes: $\chi^{2}(150)=178,84 \operatorname{com} p=0,054 ; \mathrm{CFI}=0,992 ; \mathrm{TLI}=0,989 ; \mathrm{RMSEA}=0,040 ;$ IC95\% $[0,034 ; 0,039]$.

No fator 1, que explica 43,70\% da variância, saturaram 13 itens $(11,7,10,3$, $6,2,9,12,16,5,1,8$ e 4), relacionados com a percepção dos estudantes acerca do feedback dos professores - por exemplo, item 7 ("os professores fazem perguntas que me ajudam a refletir sobre a qualidade de meu trabalho") e item 6 ("os professores dão oportunidade para eu melhorar meus trabalhos"). Os tópicos 11, 10, 3 e 15 carregam forma negativa no fator, indicando práticas de feedback menos apreciadas pelos estudantes - é o caso de 11 ("os professores dizem para fazer melhor, mas não dizem como") e 10 ("os professores fazem comentários desagradáveis ao comunicar os resultados das avaliações"). Assim, esses quatro itens foram cotados de modo inverso, de tal forma que valores mais elevados nesse fator (perto do máximo 6) indicam que os estudantes percebem que seus professores utilizam práticas eficazes de feedback, enquanto valores mais baixos (perto do mínimo 1) sinalizam que notam que seus docentes utilizam poucas práticas positivas e eficazes, sendo as ações de feedback pouco úteis. De acordo com o valor alfa de Cronbach, a consistência interna do fator é boa.

No fator 2, incluem-se seis itens $(18,17,14,19,16$ e 13$)$, que recolhem as concepções dos estudantes em relação à utilização do feedback — por exemplo, tópico 18 ("gosto de receber feedback de meus professores") e item 17 ("Eu presto atenção ao feedback de meus professores") —, explicando 5,48\% da variância. Valores mais altos nesse fator (perto de 6) indicam a percepção de uma maior utilização do feedback para o aprimoramento da aprendizagem ou do desempenho acadêmico, enquanto valores perto de 1 indicam uma percepção de menor utilização do feedback por parte dos estudantes. A consistência interna é boa.

Quanto às estatísticas descritivas, foram calculados os escores de cada fator por meio da média dos itens que o conformam. Suas respetivas estatísticas descritivas são apresentadas na Tabela 4.

$\mathrm{Na}$ Tabela 4, observa-se que os valores abarcam quase o ranking total da escala (entre 1 e 6), com médias superiores ao ponto médio da escala $(3,5)$,indicando que os estudantes percebem que seus professores apresentam práticas de feedback positivas e eficazes, além de considerar que utilizam e gostam receber esse retorno do professor. Os valores de curtose (ku) e de assimetria (sk), cerca de zero, indicam que a distribuição dos escores de cada fator apresentam uma distribuição semelhante à normal. As correlações entre os fatores são moderadas.

No que tange a relações em função do sexo, de acordo com os resultados do teste $T$ de Student, não existem diferenças significativas entre o sexo masculino e o feminino, nem na percepção dos estudantes acerca do feedback dos professores, tão pouco nas concepções em relação à utilização do feedback, conforme vemos na Tabela 5 . 


\section{Tabela 3 - Cargas fatoriais, comunalidades e estatísticas descritivas do instrumento para avaliar as concepções e percepções dos estudantes acerca do feedback dos professores (21 itens).}

\begin{tabular}{|c|c|c|c|}
\hline \multirow{2}{*}{ Itens } & \multicolumn{2}{|c|}{ Fatores } & \multirow[t]{2}{*}{ Comunalidades } \\
\hline & 1 & 2 & \\
\hline $\begin{array}{l}\text { 11. Os professores dizem para fazer melhor, mas } \\
\text { não dizem como. }\end{array}$ & $-0,829$ & 0,148 & 0,555 \\
\hline $\begin{array}{l}\text { 7. Os professores fazem perguntas que me ajudam a } \\
\text { refletir sobre a qualidade de meu trabalho. }\end{array}$ & 0,761 & 0,091 & 0,674 \\
\hline $\begin{array}{l}\text { 10. Os professores fazem comentários desagradáveis } \\
\text { ao comunicar os resultados das avaliações. }\end{array}$ & $-0,773$ & 0,220 & 0,432 \\
\hline $\begin{array}{l}\text { 3. Os comentários dos professores mostram falta de } \\
\text { respeito pelos estudantes. }\end{array}$ & $-0,748$ & 0,147 & 0,443 \\
\hline $\begin{array}{l}\text { 6. Os professores dão oportunidade para eu } \\
\text { melhorar meus trabalhos. }\end{array}$ & 0,704 & 0,081 & 0,574 \\
\hline $\begin{array}{l}\text { 2. O tom de voz e o rosto dos professores mostram } \\
\text { que acreditam que vou melhorar. }\end{array}$ & 0,688 & 0,086 & 0,555 \\
\hline $\begin{array}{l}\text { 9. Os professores explicam as formas de avaliação na } \\
\text { disciplina de maneira clara. }\end{array}$ & 0,667 & 0,055 & 0,494 \\
\hline 12. Os professores me dão um feedback confiável. & 0,645 & 0,111 & 0,518 \\
\hline $\begin{array}{l}\text { 15. Os comentários dos professores sobre meu } \\
\text { trabalho são muitas vezes difíceis de entender. }\end{array}$ & $-0,640$ & 0,098 & 0,340 \\
\hline $\begin{array}{l}\text { 5. Os professores explicam o que é esperado } \\
\text { aprender na disciplina. }\end{array}$ & 0,573 & 0,162 & 0,503 \\
\hline $\begin{array}{l}\text { 1. Os professores fazem comentários específicos } \\
\text { para me ajudar nos trabalhos. }\end{array}$ & 0,552 & 0,172 & 0,453 \\
\hline $\begin{array}{l}\text { 8. Os professores comunicam e explicam as notas a } \\
\text { cada estudante. }\end{array}$ & 0,515 & 0,064 & 0,310 \\
\hline $\begin{array}{l}\text { 4. Quando faço um trabalho, os professores } \\
\text { descrevem claramente o que não está bom. }\end{array}$ & 0,526 & 0,054 & 0,315 \\
\hline 18. Gosto de receber feedback de meus professores. & $-0,130$ & 0,864 & 0,622 \\
\hline $\begin{array}{l}\text { 17. Eu presto atenção ao feedback de } \\
\text { meus professores. }\end{array}$ & $-0,008$ & 0,799 & 0,630 \\
\hline $\begin{array}{l}\text { 14. Eu uso o feedback dos professores para } \\
\text { estabelecer metas ou objetivos para minha } \\
\text { próxima avaliação. }\end{array}$ & 0,006 & 0,752 & 0,571 \\
\hline $\begin{array}{l}\text { 19. Eu recorro ao feedback de meus professores para } \\
\text { ver o que fiz de errado. }\end{array}$ & $-0,050$ & 0,712 & 0,465 \\
\hline $\begin{array}{l}\text { 16. O feedback de meus professores faz com que eu } \\
\text { me esforce mais. }\end{array}$ & 0,222 & 0,680 & 0,700 \\
\hline $\begin{array}{l}\text { 13. Fico ansioso para obter feedback de meus } \\
\text { professores sobre meu trabalho. }\end{array}$ & $-0,166$ & 0,631 & 0,294 \\
\hline
\end{tabular}

Fonte: Banco de dados da investigação.

Elaborado pelas autoras. 
Tabela 4-Análise descritiva*.

\begin{tabular}{l|c|c}
\hline Autovalor empírico & $\mathbf{8 , 3 0}$ & $\mathbf{2 , 1 1}$ \\
\hline \% de variância & 43,70 & 5,48 \\
\hline \% de variância explicada pelos dados do estudo & 50,15 & 12,21 \\
\hline \% de variância explicada pelas AP & 11,92 & 10,92 \\
\hline Alfa & 0,89 & 0,80 \\
\hline Mínimo & 1,46 & 1,33 \\
\hline Máximo & 6,00 & 6,00 \\
\hline Média & 4,49 & 4,85 \\
\hline DP & 0,87 & 0,84 \\
\hline Sk & $-0,45$ & $-0,60$ \\
\hline Ku & $-0,03$ & 0,17 \\
\hline Correlações $(\mathrm{r})$ & & \\
\hline Fatores 1 e 2 & $0,52(\mathrm{p}<0.001)$ & \\
\hline
\end{tabular}

*Método de extração: Robust Unweighted Least Squares (RULS); método de rotação: Promin; A: baseado no minium rank factor analyses; B: calculados com base em uma matriz hipotética aleatória faturada 500 vezes para 19 itens; número $=519$. DP: desvio padrão; Sk: valor de assimetria; Ku: valor de curtose; AP: implementação ótima de análises paralelas.

Fonte: Banco de dados da investigação.

Elaborado pelas autoras.

Tabela 5 - Diferenças nas concepções e percepções sobre o feedback docente - sexo dos participantes.

\begin{tabular}{l|c|c|c|c|c|c|c}
\hline Fatores/dimensões & Sexo & $\mathrm{n}$ & $\mathrm{M}$ & $\mathrm{DP}$ & $\mathrm{T}$ & $\mathrm{df}$ & $\mathrm{p}$ \\
\hline $\begin{array}{l}\text { Percepção dos estudantes } \\
\text { acerca do feedback } \\
\text { dos professores }\end{array}$ & Feminino & 359 & 4,44 & 0,90 & $-1,73$ & 517 & 0,083 \\
\cline { 2 - 8 } & Masculino & 160 & 4,58 & 0,79 & & & \\
\hline $\begin{array}{l}\text { Concepções dos estudantes } \\
\text { em relação a própria } \\
\text { utilização do feedback }\end{array}$ & Feminino & 359 & 4,90 & 0,85 & 1,90 & 517 & 0,058 \\
\cline { 2 - 8 } & Masculino & 160 & 4,75 & 0,80 & & & \\
\hline
\end{tabular}

n: número; DP: desvio padrão; M: média; T: teste T de Student; df: graus de liberdade.

Fonte: Banco de dados da investigação.

Elaborado pelas autoras.

Quanto à idade, para compreender se existem diferenças nas percepções dos estudantes sobre o feedback docente em função da faixa etária, foi realizada uma análise de variância (Anova). Os resultados, apresentados na Tabela 6, indicam que não existem diferenças significativas na percepção do feedback docente em função da idade dos participantes.

No âmbito dos IF, foram comparadas as percepções dos estudantes em função $\mathrm{da}$ instituição que frequentavam, sendo apenas cotejados aqueles institutos em que pelo menos 30 estudantes responderam ao questionário. Os resultados, apresentados na Tabela 7 , indicam que não existem diferenças significativas entre as percepções 
Tabela 6 - Diferenças nas concepções e percepções sobre o feedback docente em função da idade dos participantes.

\begin{tabular}{l|c|c|c|c|c|c|c}
\hline Fatores/dimensões & Idade (anos) & $\mathrm{n}$ & $\mathrm{M}$ & $\mathrm{DP}$ & $\mathrm{F}$ & $\mathrm{df}$ & $\mathrm{p}$ \\
\hline \multirow{4}{*}{$\begin{array}{l}\text { Percepção dos } \\
\text { estudantes acerca do } \\
\text { feedback dos professores }\end{array}$} & 20 ou menos & 155 & 4,56 & 0,75 & 0,564 & 5,516 & 0,727 \\
\cline { 2 - 9 } & $21-25$ & 156 & 4,48 & 0,82 & & & \\
\cline { 2 - 9 } & $26-30$ & 71 & 4,39 & 1,02 & & & \\
\cline { 2 - 9 } & $31-35$ & 53 & 4,54 & 0,91 & & & \\
\cline { 2 - 9 } & $36-40$ & 33 & 4,49 & 1,03 & & & \\
\hline \multirow{4}{*}{$\begin{array}{l}\text { Concepções dos } \\
\text { estudantes em relação } \\
\text { a sua utilização } \\
\text { do feedback }\end{array}$} & 20 ou menos & 155 & 4,86 & 0,84 & 0,344 & 5,516 & 0,886 \\
\cline { 2 - 9 } & $21-25$ & 156 & 4,88 & 0,92 & & & \\
\cline { 2 - 9 } & $31-35$ & 53 & 4,77 & 0,79 & & & \\
\cline { 2 - 9 } & $36-40$ & 33 & 4,98 & 0,79 & & & \\
\cline { 2 - 9 } & Mais de 41 & 49 & 4,86 & 0,73 & & & \\
\hline
\end{tabular}

n: número; DP: desvio padrão; M: média; T: teste $T$ de Student; df: graus de liberdade.

Fonte: Banco de dados da investigação.

Elaborado pelas autoras.

Tabela 7 - Diferenças nas concepções e percepções sobre o feedback docente em função do Instituto Federal dos participantes.

\begin{tabular}{l|c|c|c|c|c|c|c}
\hline \multirow{2}{*}{ Dimensões/fatores } & $\begin{array}{c}\text { Instituto } \\
\text { Federal }\end{array}$ & $\mathrm{n}$ & $\mathrm{M}$ & $\mathrm{DP}$ & $\mathrm{F}$ & $\mathrm{df}$ & $\mathrm{p}$ \\
\hline \multirow{3}{*}{$\begin{array}{l}\text { Percepção dos } \\
\text { estudantes acerca do } \\
\text { feedback dos professores }\end{array}$} & IF $A$ & 304 & 4,49 & 0,86 & 0,35 & 3,492 & 0,790 \\
\cline { 2 - 9 } & IF $D$ & 71 & 4,39 & 0,90 & & & \\
\cline { 2 - 9 } & IF $G$ & 62 & 4,52 & 0,88 & & & \\
\hline \multirow{3}{*}{$\begin{array}{l}\text { Concepções dos } F \\
\text { estudantes em relação a } \\
\text { sua utilização do feedback }\end{array}$} & IF $A$ & 304 & 4,94 & 0,84 & 2,59 & 3,492 & 0,052 \\
\cline { 2 - 9 } & IF $D$ & 71 & 4,84 & 0,76 & & & \\
\cline { 2 - 9 } & IF $G$ & 62 & 4,78 & 0,79 & & & \\
\hline
\end{tabular}

IF: Instituto Federal; n: número; DP: desvio padrão; M: média; T: teste $T$ de Student; df: graus de liberdade.

Fonte: Banco de dados da investigação.

Elaborado pelas autoras.

dos estudantes que frequentam cursos nos Institutos A (Centro-Oeste), D (Sul), $\mathrm{G}$ (Norte) e F (Sul).

Utilizando os fatores identificados como variáveis manifestas, foi, ainda, estimado um modelo causal da variável latente "percepções e concepções do feedback do estudante" sobre a variável latente "desempenho acadêmico", estimado por meio das variáveis 
manifestas pela proporção do número de disciplinas totais já reprovadas e pela média autodeclarada das notas dos estudantes do semestre letivo (1\%/2019). Para tal, utilizou-se o software AMOS (vs. 25). Tal questão pode ser observada no modelo na Figura 1.

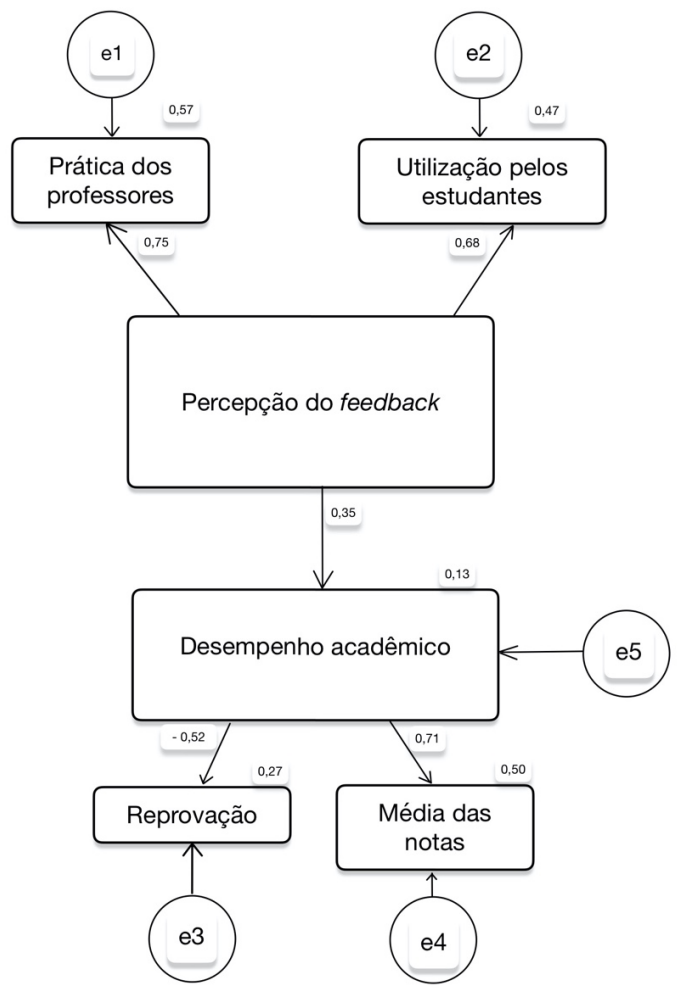

Fonte: Elaborado pelas autoras.

e1-e5: parte da variância das variáveis que não são explicadas pela medida utilizada (e1, e2, e3 e e4) ou pelo modelo apresentado $(\mathrm{e} 5) \cdot \chi^{2}(1)=0,49 ; \mathrm{p}=0,486 ; \mathrm{CFI}=1,00 ; \mathrm{TLI}=1,02 ; \mathrm{RMSEA}=0,000$, intervalo de confiança $95 \%=[0,000 ; 0,092]$.

Figura 1 - Modelo causal das percepções e concepções do feedback do estudante sobre o desempenho acadêmico.

De acordo com a Figura 1, todas as variáveis manifestas contribuem significativamente em sua variável latente $(\lambda<|0,50|)$. A trajetória entre as "percepções e concepções do feedback do estudante" o o "desempenho acadêmico" foi significativa $(b=0,542 ; \beta=0,35 ; p<0,001)$, sendo que as percepções e concepções do feedback do estudante explicaram $13 \%$ do desempenho acadêmico. $\mathrm{O}$ modelo revelou uma qualidade de ajustamento excelente: $\chi^{2}(1)=0,49 \operatorname{com} p=0,486 ; \mathrm{CFI}=1,00 ; \mathrm{TLI}=$ 1,$02 ;$ RMSEA = 0,000; IC95\% [0,000; 0,092].

\section{CONSIDERAÇÕES FINAIS}

A elaboração da presente escala partiu da adaptação de outros dois instrumentos, a SCoF III e a escala de percepção dos alunos sobre o feedback dos professores, con- 
cordando com a orientação dos autores de explorar a utilização dos instrumentos em investigações futuras (Carvalho et al., 2014; Brown, Peterson e Yao, 2016). Os resultados deste estudo permitem verificar, primeiramente, que o instrumento elaborado apresenta qualidade psicométrica de validade e de fidelidade para os participantes da presente investigação. Esta versão possibilitará novas análises, além de, igualmente, incentivar novos estudos exploratórios e confirmatórios com estudantes de ensino superior.

Verificou-se que os estudantes apreciam receber feedback eficaz do professor, considerando-o útil ao desempenho acadêmico. Os resultados mostram que, quanto maior a percepção de uma utilização de práticas eficazes de feedback docente, maior o apreço delas por parte dos estudantes do ensino superior e melhor o desempenho do discente. Isto é, as notas são mais altas (pois relacionam-se positivamente com o desempenho acadêmico; o valor do peso fatorial é 0,71 ) e menor é o índice de reprovação (porque este se relaciona negativamente com o desempenho; o valor do peso fatorial é -0,52). Esse resultado vai ao encontro da literatura (Pereira e Flores, 2013; Farias et al., 2015; Silva et al., 2019), indicando que os estudantes tendem a valorizar o feedback de seus professores, no sentido de uma crítica construtiva para além de meras classificações. Isso é coerente com a ideia de que o feedback possui uma importante influência na aprendizagem e no desempenho do aluno (Hattie, 1992; Mory, 2004; Hattie e Timperley, 2007; Brookhart, 2008; Carvalho et al., 2014).

Este estudo contribui, assim, para a compreensão e a percepção do feedback do professor na perspectiva discente, quanto ao fator de aprendizagem no ensino superior, instrumentalizando acadêmicos, professores e gestores em futuras investigações, práticas, políticas e projetos. Reconhecer a importância do feedback docente e entender que os discentes apreciam recebê-lo e o consideram útil ao processo acadêmico pode levar os professores a (re)pensar práticas, visando a ir além de classificações, do que está correto ou errado e da dimensão descritiva na própria prática de feedback.

Quanto a relações de gênero, idade e instituição de ensino, não foram encontradas correlações significativas. A ausência de diferenciação quanto à instituição sugere um alinhamento e uma coerência dentro da própria Rede de Educação Profissional e Tecnológica, pois os Institutos Federais, ainda que em regiões tão distintas, como o Norte, o Centro-Oeste e o Sul do Brasil, apresentam, basicamente, a mesma estrutura em questões administrativas, objetivos estratégicos e formas de acesso.

Com este estudo, visa-se a contribuir com a redução da lacuna acadêmica e literária existente no que tange a estudos no Brasil sobre a temática, especialmente no âmbito de Institutos Federais de Educação, Ciência e Tecnologia enquanto instituições de ensino superior público, gratuito e de qualidade. Os resultados encontrados no presente trabalho urgem para que professores trabalhem com os estudantes na construção e na aplicabilidade de estratégias de feedback, visando à aprendizagem. Com isso, deve-se oportunizar diálogos e reflexão sobre o que se aprendeu e sobre o que ainda falta aprender. O feedback dos professores é, portanto, um elemento que permite aprimorar a aprendizagem dos alunos, apresentando relação com o sucesso acadêmico. Assim, pensar contributos para um processo de ensino-aprendizagem no ensino superior implica voltar-se para a dimensão pedagógica do ensino e (re)criar práticas de feedback nas quais os estudantes sejam ouvidos e atendidos. 


\section{REFERÊNCIAS}

ASKEW, S.; LODGE, C. Gifts, ping-pong and loops: linking feedback and learning. In: ASKEW, S. Feedback for learning. London: Routledge; Falmer, 2000. p. 1-17.

ASÚN, R.A.; RDZ-NAVARRO, K.; ALVARADO,J.M.Developing Multidimensional Likert Scales Using Item Factor Analysis: The Case of Four-point Items. Sociological Methods e Research, Santiago, v. 45, n. 1, p. 109-133, jan. 2015. https://doi. org/10.1177\%2F0049124114566716

BRANDT, J. Z. Ensino superior no Brasil: fatores influenciadores no desempenho acadêmico dos alunos de graduação. Braga: Universidade do Minho, 2017.

BRANDT,J.Z.; TEJEDO-ROMERO, F.; ARAUJO, J. F. F. E. Fatores influenciadores do desempenho acadêmico na graduação em administração pública. Educação e Pesquisa, São Paulo, v. 46, p. 1-20,jan. 2020. https://doi.org/10.1590/S1678-4634202046202500 BRASIL. Lei no 11.892, de 29 de dezembro de 2008. Institui a Rede Federal de Educação Profissional, Científica e Tecnológica, cria os Institutos Federais de Educação, Ciência e Tecnologia, e dá outras providências. Diário Oficial da União, Brasília, 30 dez.2008. Disponível em: http://www.planalto.gov.br/ccivil_03/_ato2007-2010/2008/ lei/111892.htm. Acesso em: 20 nov. 2020.

BROOKHART, S. M. How to give effective feedback to your students. Alexandria: Association for Supervision and Curriculum Development, 2008.

BROWN, G.T. L.; PETERSON E. R.; YAO, E. S. Student conceptions of feedback: Impact on self-regulation, self-efficacy, and academic achievement. British Journal of Educational Psychology, Leicester, v. 86, n. 4, p. 606-629, dez. 2016. https://doi. org/10.1111/bjep.12126

BUTLER,D. L.; WINNE, P.H. Feedback and Self-Regulated Learning: A Theoretical Synthesis. Review of Educational Research, Thousand Oaks, v. 65, n. 3, p. 245-281, set. 1995. https://doi.org/10.3102\%2F00346543065003245

CARVALHO, C.; CONBOY, J.; SANTOS, F.; TAVARES, D.; MARTINS, D.; SALEMA,H.; FIUZA, E.; GAMA, A. Escala de percepção dos alunos sobre o feedback dos professores: construção e validação. Laboratório de Psicologia, Lisboa, v. 12, n. 2, p. 113-124, 2014. http://doi.org/10.14417/lp.880

CARVER, M. Exploring students' concepts of feedback as articulated in large-scale surveys: a useful proxy and some encouraging nuances. Practitioner Research in Higher Education, Carlisle, v. 10, n. 1, p. 39-52, 2016. Disponível em: http://hdl.handle. net/10023/12727. Acesso em: nov. 2020.

COLLINS, J. Education techniques for lifelong learning: principles of adult learning. RadioGraphics, Oak Brook, v. 24, n. 5, p. 1483-1489, 2004. https://doi.org/10.1148/ rg.245045020

DURÁN SANTOMIL, P.; MASIDE SANFIZ, J. M.; RODEIRO PAZOS, D.; CANTORNA AGRA, S. Determinantes del rendimiento académico del alumnado de una asignatura de Contabilidad: el caso de la USC. Revista de Docencia Universitaria, Valencia, v. 14, n. 1, p. 151-178, 2016. Disponível em: https://dialnet.unirioja.es/ descarga/articulo/5741998.pdf. Acesso em: 20 nov. 2020. 
EVANS, C.; WARING, M. A consideration of physical education student teachers' feedback seeking behavior's from a cognitive styles perspective. In: annual international conference of the European learning styles information network: exploring styles to enhance learning and teaching in diverse contexts? Aveiro. Proceedings [...]. Aveiro: Departamento de Educação da Universidade de Aveiro, 2010. p. 182-184.

FARIAS, C.; CARDOSO, B.; NETO, E.; CARVALHO, R; CURTIS, D. Feedback no processo de aprendizagem: percepção dos estudantes de Odontologia em uma universidade brasileira. Revista da ABENO, Brasília, v. 15, n. 3, p. 35-42, out. 2015. https://doi.org/10.30979/rev.abeno.v15i3.160

FERNANDES, D. Avaliação das aprendizagens: desafios às teorias, práticas e políticas. Lisboa: Texto Editores, 2005.

FERRANDO, P.J.; LORENZO-SEVA, U. FACTOR: A computer program to fit the exploratory factor analysis model. Behavior Research Methods, Chicago, v. 38, n. 1, p. 88-91, fev. 2006. https://doi.org/10.3758/BF03192753

FERRANDO,P.J.; LORENZO-SEVA,U.Factor 9.2: A comprehensive program for fitting exploratory and semiconfirmatory factor analysis and IRT models. Applied Psychological Measurement,v.37,n.6,p.497-498,mai.2013.https://doi.org/10.1177\%2F0146621613487794 FERRANDO, P. J.; LORENZO-SEVA, U. On the Added Value of Multiple Factor Score Estimates in Essentially Unidimensional Models. Educational and Psychological Measurement, Washington, v. 79, n. 2, p. 249-271, mai. 2019. https:// doi.org/10.1177\%2F0013164418773851

FERREIRA,M.P.M. Teoria(s) da atribuição: um quadro explicativo para o rendimento acadêmico. Revista Brasileira de Educação, Rio de Janeiro, v. 24, ago. 2019. https:// doi.org/10.1590/S1413-24782019240037

FLORES, M. A.; VEIGA SIMÃO, A. M.; BARROS, A.; PEREIRA, D. Perceptions of effectiveness, fairness and feedback of assessment methods: a study in higher education. Studies in Higher Education, London, v. 40, n. 9, p. 1.523-1.534, abr. 2014. https:// doi.org/10.1080/03075079.2014.881348

HATTIE, J. Measuring the Effects of Schooling. Australian Journal of Education, [Internet],v.36,n.1, p.5-13, abr.1992. https://doi.org/10.1177\%2F000494419203600102 HATTIE, J.; TIMPERLEY, H. The Power of Feedback. Review of Educational Research, Thousand Oaks, v. 77, n. 1, p. 81-112, mar. 2007. https://doi. org/10.3102\%2F003465430298487

HOLGADO-TELLO, F. P.; CHACÓN-MOSCOSO, S.; BARBERO-GARCÍA, I.; VILA-ABAD, E. Polychoric versus Pearson correlations in exploratory and confirmatory factor analysis of ordinal variables. Quality e Quantity, v. 44, n. 1, p. 153-166, set. 2010. https://doi.org/10.1007/s11135-008-9190-y

HOWARD, M. A Review of Exploratory Factor Analysis Decisions and Overview of Current Practices: What We Are Doing and How Can We Improve? International Journal of Human-Computer Interaction, [Internet], v. 32, n.1, p. 51-62, 2016. https:// doi.org/10.1080/10447318.2015.1087664

IRVING, S. E.; PETERSON, E. R. Conceptions of feedback (CoF) inventory (Version 2). Auckland: University of Auckland, 2006. 
KUENZER, A. Exclusão includente e inclusão excludente: a nova forma de dualidade estrutural que objetiva as novas relações entre educação e trabalho. In: SAVIANI, D.; SANFELICE, J. L.; LOBARDI, J. C. Capitalismo, trabalho e educação. Campinas: Autores Associados, 2005. p. 77-96.

LORENZO-SEVA, U.; VAN GINKEL, J. R. Multiple Imputation of missing values in exploratory factor analysis of multidimensional scales: estimating latent trait scores. Anales de Psicología/Annals of Psychology, Murcia, v. 32, n. 2, p. 596-608, abr. 2016. http://doi.org/10.6018/analesps.32.2.215161

MARÔCO, J. P. Análise Estatística com o SPSS Statistics. 6. ed. Pêro Pinheiro: ReportNumber, 2014.

MATOS, D. A avaliação no ensino superior: concepções múltiplas de estudantes brasileiros. 2010. 139 f. Tese (Doutorado em Psicologia) - Universidade Federal de Minas Gerais, Belo Horizonte, 2010.

MATOS, D.; BROWN, G. T. L. Comparing university students' conceptions of assessment: Brazilian and New Zealand beliefs. In: CARVALHO, C.; CONBOY J. (Ed.). Feedback, identidade, trajetórias escolares: dinâmicas e consequências. Lisboa: IE-Ulisboa, 2015. p. 177-194.

MINGHELLI, M. A nova estrutura normativa de ciência, tecnologia e inovação no Brasil. Encontros Bibli, Florianópolis, v. 23, n. esp. 1, p. 143-151, 2018. https://doi. org/10.5007/1518-2924.2018v23nespp143

MONTEIRO, V.; MATA, L.; SANTOS, N.; SANCHES, C.; GOMES, M. Classroom Talk: The Ubiquity of Feedback. Frontiers in Education, Lausanne, v. 4, p. 140-158, dez. 2019. https://doi.org/10.3389/feduc.2019.00140

MORY, E. H. Feedback research revisited. In: SPECTOR, J. M.; MERRILL, M. D.; ELLEN, J.; BISHOP, M. J. (Ed.). Handbook of research on educational communications and technology. Vol. 2. New York: Springer, 2004. p. 745-783.

PEREIRA,D. R.; FLORES, M.A. Avaliação e feedback no ensino superior: um estudo na Universidade do Minho. Revista Iberoamericana de Educación Superior, Huixquilucan, v. 4, n. 10, p. 40-54, 2013. https://doi.org/10.1016/S2007-2872(13)71923-1

PEREIRA, D.; FLORES,M.A.; VEIGA SIMÃO,A.M.; BARROS, A.Effectiveness and relevance of feedback in Higher Education: A study of undergraduate students. Studies in Educational Evaluation, v. 49,p.7-14,2016. http://doi.org/10.1016/j.stueduc.2016.03.004 SADLER, D. R. Beyond feedback: Developing student capability in complex appraisal. Assessment \& Evaluation in Higher Education, [Internet], v. 35, n. 5, p. 535-550, ago. 2010. https://doi.org/10.1080/02602930903541015

SADLER, D. R. Formative Assessment: Revisiting the Territory. Assessment in Education: Principles, Policy e Pratice, [Internet], v. 5, n. 1, p. 77-84,jul. 2016. https:// doi.org/10.1080/0969595980050104

SCHREIBER, J.; NORA, A.; STAGE, F.; BARLOW, L.; KING, J. Confirmatory Factor Analyses and Structural Equations Modeling: An Introduction and Review. Journal of Educational Research, v. 99, n. 6, p. 323-338, ago. 2006. https:// doi.org/10.3200/JOER.99.6.323-338 
SILVA, J.; CARVALHO, C.; ALMEIDA, C.; FREIRE, S. Vozes dos alunos sobre feedback: um estudo no ensino superior. In: MONTEIRO, V.; MATA, L.; MARTINS, M.A.; MORGADO,J.; SILVA, J. G.; SILVA, A. C.; GOMES, M. (orgs.). Educar hoje: diálogos entre psicologia, educação e currículo. Lisboa: Edições ISPA, 2019. p. 325-331. SWAFFIELD, S. Feedback: The central process in assessment for learning. In: SWAFFIELD, S. (Ed.). Unlocking assessment: understanding for reflection and application. Oxon, UK: Routledge, 2008. p. 57-72.

TIMMERMAN, M.E.; LORENZO-SEVA,U.Dimensionality assessment of ordered polytomous items with parallel analysis. Psychological Methods, v. 16, n. 2, p. 209-220, jun. 2011. https://doi.org/10.1037/a0023353

VARGAS, G. M. G. Factores asociados al rendimiento académico tomando en cuenta el nível socioeconómico: estudio de regresion múltiple en estudiantes universitários. Revista Electrónica Educare, Heredia, v. 18, n. 1, p. 119-154, abr. 2014. http://doi. org/10.15359/ree.18-1.6

\section{SOBRE AS AUTORAS}

Juana de Carvalho Ramos Silva é doutoranda em psicologia da educação pela Universidade de Lisboa (Portugal). Professora do Instituto Federal de Educação Ciência e Tecnologia de Brasília (IFB).

E-mail: juanacarvalho@gmail.com

Carolina Fernandes Carvalho é doutora em psicologia da educação pela Universidade de Lisboa (Portugal). Professora da mesma instituição. E-mail: cfcarvalho@ie.ulisboa.pt

Conflitos de interesse: As autoras declaram que não possuem nenhum interesse comercial ou associativo que represente conflito de interesses em relação ao manuscrito.

Financiamento: $\mathrm{O}$ estudo não recebeu financiamento.

Contribuições das autoras: Administração do Projeto, Análise Formal, Conceituação, Curadoria de Dados, Escrita - Primeira Redação: Silva, J.C.R.; Carvalho, C.F. Revisão e Edição: Silva, J.C.R.; Carvalho, C.F. 